\title{
A Method for Restoring the Uniqueness of Temperature and Its Application to the Malthus-Verhulst Equation with a Stochastic Term
}

\author{
Yu. V. Bibik ${ }^{1}$ \\ ${ }^{1}$ Dorodnicyn Computing Centre of Russian Academy of Sciences, Moscow, Russia \\ Correspondence: Yu. V. Bibik, Dorodnicyn Computing Centre of Russian Academy of Sciences, Vavilov st. 40, \\ Moscow 119333, Russia. E-mail: yvbibik@ccas.ru
}

\author{
Received: June 13, 2012 Accepted: November 28, 2012 Online Published: January 21, 2013 \\ doi:10.5539/jmr.v5n1p65 URL: http://dx.doi.org/10.5539/jmr.v5n1p65
}

\begin{abstract}
A version of the renormalization group (renormgroup) method is developed, which is called the method for restoring the uniqueness of temperature. This method is applied to the Malthus-Verhulst equation with a stochastic term. This equation from mathematical biology is reduced to a quantum field problem for the one-dimensional case.

To establish the dependence of the temperature of the stochastic term on the scale of the block-spin variables, the problem is renormalized using the quantum field renormgroup method (the Wilson technique and the minimal subtraction scheme).

As a result of renormalization, the dependence of the temperature of the stochastic term on the scale of the blockspin variables turns out to be the same but ambiguous in both cases.

To resolve this difficulty, a special procedure for restoring the uniqueness of the temperature dependence is developed; this procedure makes it possible to determine the dependence of the stochastic term temperature on the scale of the block-spin variables and calculate the correlation length.
\end{abstract}

Keywords: renormalization group, Malthus-Verhulst equation, stochastic dynamics, block-spin variables

\section{Introduction}

In this paper, we investigate the possibility to apply the quantum field theory techniques to a mathematical biology equation. As the model, we use the Malthus-Verhulst equation with a stochastic term.

In its modern form, mathematical biology originates in the classical work of the famous English demographer and political economist Thomas Robert Malthus called An Essay on the Principle of Population (1798). To describe the dynamics of the population growth, Malthus proposed a population dynamics equation, which describes the exponential growth of population. This equation does not take into account self-restriction processes; for that reason it yields an exponential growth of population.

The Malthus equation was elaborated by Verhulst in 1838. He took into account the self-restriction effects related to the competition for the environment conditions, which restrict the population growth.

Later, the development of mathematical biology equations followed the path of taking into account a greater number of species.

Lotka (1925) and Volterra (1926) proposed equations for describing the simultaneous dynamics of two speciespredator and prey. These equations were given the name of Lotka-Volterra equations. Later, the so-called Volterra chain was proposed, which takes into account an arbitrary number of species in the food chain, where each species is a predator with respect to the preceding species and a prey with respect to the next one. A common drawback of these models is that they ignore habitat random factors.

To overcome this drawback, Kerner $(1957,1959)$ used statistical simulation of biological processes. To take into account a specific configuration of species, he used the Boltzmann factor and applied the statistical sum method.

In this paper, another approach to inclusion of the habitat random factors is used-a stochastic term is added to the original Malthus-Verhulst equation. This term should take into account how the species in question is affected by 
other species in the environment.

The study of the original equation is important because it is a simple realistic equation of mathematical biology that accounts for habitat random factors. This is important because the population dynamics is not smooth but undergoes random oscillations due to natural social and ecological factors. This is taken into account by the stochastic term added to the Malthus-Verhulst equation.

The use of the stochastic term is not a novelty. For example, it was used in the Langevin equation describing the Brownian motion (1908).

The introduction of the stochastic term into the equation under examination is important not only from the biological point of view. It opens wide mathematical possibilities and allows one to apply quantum field techniques to study the equation in question.

Thus, this paper aims at applying quantum field techniques to the equation under examination, reveal the associated difficulties and find out how they can be mitigated.

The Malthus-Verhulst equation with a stochastic term is a nonlinear evolutionary equation. The application of quantum field techniques and, specifically, the application of the renormgroup method to nonlinear evolutionary equations is not new. This method is used, for example, in fluid dynamics for the study of turbulence for NavierStokes equations with a stochastic force (Adzhemyan, Antonov, \& Vasil'ev, 1996).

In this paper, we use the concepts of renormalization group, block-spin variables and running coupling constants for the transformation of the model of nonlinear equation with a stochastic term into the quantum field model.

Here, these concepts are briefly described.

The renormalization group is a powerful tool for the study of all systems possessing scale invariance. It was first proposed by Gell-Mann and Low (1954) to improve the series in perturbation theory in quantum field theory and by Wilson (1971 \& 1974) to study critical phenomena in statistical physics. With the emergence of deterministic chaos theory, the functional group method was developed by Feigenbaum (1978 \& 1979) and others.

Block-spin variables represent a degree of freedom of the system under examination constructed from the set of degrees of freedom by integrating over them. The scale of the block-spin variables is determined by the number of degrees of freedom included. The block-spin variables are most clearly defined in the theory of magnetics, where they are the sum over a finite number of spins. By analogy, they can be defined for systems with the continuum of degrees of freedom as is the case in this paper or, for example, in field theory $\varphi-4$. In this paper, an interesting problem of the relationship of the scale of block-spin variables with the temperature of the stochastic term is formulated.

The running coupling constants are a key object in field theory; they determine the intensity of interaction between particles depending on the distance between them. In this paper, the running coupling constants are represented in terms of the stochastic term temperature.

The main assumption of this paper is that the application of quantum field techniques to the equation in question will make it possible to relate the scale of block-spin variables to the temperature of the stochastic term. This assumption is verified using the renormalization group method. This method relates the scale of lengths or momenta to the magnitude of the running coupling constants. In the case considered in this paper, the role of the running coupling constants is played by the temperature of the stochastic term.

The relation between the scale of block-spin variables and the magnitude of the running coupling constants is most clearly determined by the Wilson technique. In this method, the integration over the extra degrees of freedom is performed directly, and the change in the running coupling constants is analyzed.

However, the basic technique used to determine the running coupling constants is the minimal subtraction (MS) scheme (Ramond, 1981; Collins, 1984). In this method, the normalizing momentum is used instead of the scale of the block-spin variables, which is less intuitively clear. However, this method has an unquestionable advantage-the compactness of calculations in the one-dimensional case.

We failed to do with the Wilson technique and the MS scheme only because two running coupling constants $T$ and $T^{\frac{1}{2}}$ appear when the original equation is transformed into a quantum field problem, and each of them depends on the scale of the block-spin variables in a specific way. However, the stochastic term has only one temperature, 
and its dependence on the scale of the block-spin variables must be unambiguous. Hence, we need to develop a procedure for restoring the unique dependence of the temperature on the scale of the block-spin variables. The development of such a procedure is a part of this paper. It is called the procedure for restoring the uniqueness of temperature, and it is described in Subsection 2.6.

As a result, the dependence of the stochastic term temperature on the scale of the block-spin variables is established.

\section{Investigation Techniques}

\subsection{Salient Features of the Method}

This method consists of three parts.

The first part assumes the transition from the Malthus-Verhulst equation with a stochastic term, which describes the chaotic dynamics, to a problem in quantum field theory.

The introduction of the stochastic term $\eta$ indirectly takes into account the interaction of the biological species described in the equation with other species and the influence of various factors (such as seasonality) on its dynamics Basykin, 1998; Bibik \& Sarancha, 2008). The use of the stochastic term $\eta$ offers a twofold benefit-it makes it possible to take into account additional biological factors and to reduce the problem to a field theory problem.

The second part is the renormalization of this problem using two methods (the MS scheme and the Wilson technique) to compare the results and make the basic principles of the proposed method clear.

The third part is the development of a procedure for restoring the unique dependence of the stochastic term temperature on the scale of the block-spin variables and determining this dependence. The procedure is developed to overcome the difficulty arising when the renormalization is done using two methods-in the course of renormalization by the Wilson technique and by the MS scheme, two running coupling constants appear, while there is only one temperature.

The composition of the method is illustrated in the following block diagram.

\begin{tabular}{|l|l|}
\hline $\begin{array}{ll}\text { Chaotic dynamics } \\
\phi_{t}=Q(\phi)+\eta(t), \text { where } \eta(t) \\
\text { is the stochastic term }\end{array}$ & $\begin{array}{l}\text { Quantum field theory } \\
Z=\int[d \phi] e^{-\frac{1}{2 \sigma^{2}} \int d t\left(\phi_{t}-Q(\phi)^{2}\right.} \\
\text { (Wilson technique, MS scheme, } \\
\text { determination of the running coupling } \\
\text { constants) }\end{array}$ \\
$\qquad \begin{array}{l}\text { Procedure for restoring the uniqueness } \\
\text { of temperature, determination of the } \\
\text { dependence of the stochastic term } \\
\text { temperature on the scale of the block- } \\
\text { spin variables. }\end{array}$ \\
\hline
\end{tabular}

\subsection{Methodology of the Study}

In the development of the first part of the method, the classical problem with the stochastic term is replaced by a problem in quantum field theory. This transition is original but not new. In particular, it was used in the surface growth problem described by the Kardar-Parisi-Zhang equation (Kardar, Parisi, \& Zhang, 1986), and it is described in detail in the book by Zee (2003).

The method is based on functional integration with respect to the stochastic term $\eta(t)$ in the formal expression for the statistical sum.

In the second part of the method, upon the transition to the quantum field problem, the renormgroup methods are used (the MS scheme (Ramond, 1981; Collins, 1984) and the Wilson technique (Wilson, 1971; Wilson \& Kogut, 1974)). 
In the case under examination, the main objects under study are the temperature of the stochastic term, which play the role of the running coupling constants, and the block-spin variables. To determine these variables, intervals (cells) of length $L$ are defined on the time axis $t$, and the field $\varphi$ is integrated over these intervals.

The methodology of the study takes into account the fact that the system of nonlinear equations with stochastic terms depends only on time. This is extremely important because such dependence yields a one-dimensional quantum field problem. This problem then becomes a quantum mechanics problem, which considerably facilitates the investigation.

There are no divergences when Green's function is calculated. Therefore, there is no need to consider counterterms because they do not appear in the Lagrangian in the one-dimensional case. The MS scheme becomes almost trivial in this case, which is used in the paper.

In the third part of the method, the novel methodology proposed by the author of this paper is used. It is applied for restoring the uniqueness of temperature and for determining the dependence of the stochastic term temperature on the scale of the block-spin variables. This methodology is thoroughly described in Subsection 2.6.

The analysis is divided into steps of which each is thoroughly described in the following subsections:

- The transition from the Malthus-Verhulst equation with a stochastic term to the quantum field problem is described in Subsection 2.3.

- The renormalization of the running coupling constants by the Wilson techniques is described in Subsection 2.4.

- The renormalization of the running coupling constants by the MS scheme in the one-dimensional case is described in Subsection 2.5.

- The restoration of the unique dependence of the stochastic term temperature on the scale of the block-spin variables in Subsection 2.6.

The results are presented in Section 3.

\subsection{Transition from the Malthus-Verhulst Equation with a Stochastic Term to the Quantum Field Problem}

With account for the stochastic term, the Malthus-Verhulst equation takes the form

$$
\phi_{t}=\gamma \phi(K-\phi)+\eta(t)
$$

The stochastic term $\eta(t)$ is the Gaussian noise, which satisfies the condition

$$
P(\eta)=C e^{-\frac{1}{2 \sigma^{2}} \int d t \eta(t)^{2}} .
$$

Here, we can apply the technique for changing from a dynamics problem to a problem in quantum field theory, which is thoroughly described in the book by Zee (2003). As a result, we replace the mathematical biology equation with a quantum field problem.

The transition is performed using the formal representation of the statistical sum $Z$ and the subsequent integration with respect to $\eta$.

By definition, the statistical sum for Equation (2.3.1) is

$$
Z=\int d \phi \int d \eta e^{-\frac{1}{2 \sigma^{2}} \int \eta^{2} d t} \delta\left(\phi_{t}-\gamma \phi(K-\phi)-\eta\right)
$$

Here, by the expression $\delta\left(\phi_{t}-\gamma \phi(K-\phi)-\eta\right)$ we mean the construct

$$
\delta\left(\phi_{t}-\phi(1-\phi)-\eta\right)=\lim _{h \rightarrow 0} \prod_{n=-\infty}^{\infty} \delta\left(\frac{\phi(n+1) h-\phi(n h)}{h}-\gamma \phi(n h)(K-\phi(n h))-\eta(n h)\right) .
$$

The use of the $\delta$ function allows us to represent the dynamics equation in the probabilistic language.

Integrating with respect to $\eta$ in the representation of the statistical $\operatorname{sum} Z$, we obtain

$$
Z=\int d \phi e^{-S^{\prime}(\phi)}
$$


Here, the Lagrangian is

$$
S(\phi)=\frac{1}{2 \sigma^{2}} \int d t\left[\phi_{t}-\gamma \phi(K-\phi)\right]^{2} .
$$

We denote $\frac{1}{2 \sigma^{2}}$ by $\beta$. Here, $\beta$ is the reciprocal of temperature. Then, the Lagrangian takes the form

$$
S(\phi)=\beta \int d t\left[\phi_{t}-\gamma \phi(K-\phi)\right]^{2}
$$

or

$$
S(\phi)=\beta \int d t\left(\phi_{t}^{2}-2 \gamma K \phi_{t} \phi+\gamma^{2} K^{2} \phi^{2}+2 \gamma \phi_{t} \phi^{2}-2 \gamma^{2} K \phi^{3}+\gamma^{2} \phi^{4}\right) .
$$

The terms $2 \gamma K \phi_{t} \phi$ and $2 \gamma \phi_{t} \phi^{2}$ are total derivatives, and they can be dropped.

If we worked with the Lotka-Volterra equations with stochastic terms, expressions of the form $\phi_{t} \phi \psi$ would emerge in the interaction Lagrangian, and we would not be able to get rid of them, which would make the analysis much more difficult.

This is one of the advantages of choosing the Malthus-Verhulst equation as a model. As a result, we obtain

$$
S(\phi)=\beta \int d t\left(\phi_{t}^{2}+\gamma^{2} K^{2} \phi^{2}-2 \gamma^{2} K \phi^{3}+\gamma^{2} \phi^{4}\right) .
$$

Now, we want to turn the reciprocal temperature $\beta$ into the running coupling constants $\lambda$ and $g$. We have two constants because there are two interaction terms $\phi^{3}$ and $\phi^{4}$. Here is the root of the future difficulties. The running constants $\lambda$ and $g$ give two different dependences of the reciprocal temperature $\beta$ on $\mu=\frac{1}{L}$. To resolve this difficulty, the author of this paper proposes a temperature uniqueness restoration procedure. To transform the reciprocal temperature $\beta$ into the running coupling constants, we make the change of variables $\beta \phi^{2} \rightarrow \phi^{2}$. Then, we obtain the Lagrangian in the form

$$
S(\phi)=\int d t\left(\phi_{t}^{2}+\gamma^{2} K^{2} \phi^{2}-\frac{2 \gamma^{2} K}{\sqrt{\beta}} \phi^{3}+\frac{\gamma^{2}}{\beta} \phi^{4}\right) .
$$

For convenience, we make one more change of variables

$$
\phi \rightarrow \frac{\phi}{\sqrt{2}}
$$

Then, the Lagrangian takes the form

$$
S(\phi)=\int d t\left[\frac{\phi_{t}^{2}}{2}+\frac{\gamma^{2} K^{2} \phi^{2}}{2}-\frac{\gamma^{2} K \lambda \phi^{3}}{\sqrt{2}}+\frac{\gamma^{2} g \phi^{4}}{4}\right]
$$

with the more familiar quadratic right-hand side, where the coupling constants are

$$
\lambda=\frac{1}{\sqrt{\beta}} \text { and } g=\frac{1}{\beta} \text {. }
$$

We can make the change of variables $\phi \rightarrow-\phi$ in formula (2.3.12) and use the action

$$
S(\phi)=\int d t\left[\frac{\phi_{t}^{2}}{2}+\frac{\gamma^{2} K^{2} \phi^{2}}{2}+\frac{\gamma^{2} K \lambda \phi^{3}}{\sqrt{2}}+\frac{\gamma^{2} g \phi^{4}}{4}\right] .
$$

Note that the temperature corresponding to the stochastic term is $T=\frac{1}{\beta}$.

In the transition to the problem in quantum field theory, the stochastic term temperature was transformed into two coupling constants $\lambda=\sqrt{T}$ and $g=T$.

Thus, we reduced the Malthus-Verhulst equation with stochastic term (2.3.1) to the quantum field problem with Lagrangian (2.3.14).

Next, we determine the relation between the scale of the block-spin variables and the temperature. This relation is difficult to determine for the original Malthus-Verhulst equation with the stochastic term. For that reason, we determine it using the quantum field problem. 
There is a similar problem in field theory. The point is that the field theory is valid not for all arbitrarily large energies. Beyond a certain threshold, the phenomena must be described by other theories; however, we have no clear value for this scale. In this case, the following approach can be used.

On the one hand, the cutoff by the scale of the imparted momenta is introduced into the expression for the statistical sum $Z$. On the other hand, the coupling constants are fitted such that the resultant value of the statistical sum $Z$ remains unchanged.

Here, we do in the same fashion. We introduce the cutoff by the scale of the imparted momenta $\Lambda$, and fit the coupling constants as required.

Then, the statistical sum $Z$ takes the form

$$
Z=\int[d \phi]_{\Lambda} e^{-\int d t S(\phi, \lambda(\Lambda), g(\Lambda))},
$$

where the measure for functional integration is

$$
[d \phi]_{\Lambda}=\prod_{|k|<\Lambda} d \phi(k) .
$$

Next, we determine the dependence of the running coupling constants $\lambda(\Lambda)$ and $g(\Lambda)$ on the scale of the momenta cutoff and on the scale of the block-spin variables. In this case, $\Lambda=\frac{1}{L}$, where $\mathrm{L}$ is the scale of the block-spin variables. For that purpose, we use the Wilson technique and the MS scheme. First, we renormalize the running coupling constants using the Wilson technique.

\subsection{Renormalization of the Running Coupling Constants Using the Wilson Technique}

To determine the dependence of the running coupling constants $\lambda(\Lambda)$ and $g(\Lambda)$ on the scale of the momenta cutoff, we use a renormgroup method called the Wilson technique.

In this method, we use the dimensional coupling constants $\lambda$ and $g$ and the dimensionless momenta scale $b$.

Using the cutoff by momenta, we write statistical sum (2.3.15) in the form

$$
Z=\int[d \phi]_{\Lambda} \exp \left(-\int d^{d} x\left[\frac{1}{2}\left(\partial_{\mu} \phi\right)^{2}+\frac{m^{2}}{2} \phi^{2}+\frac{\gamma^{2} K \lambda}{\sqrt{2}} \phi^{3}+\frac{\gamma^{2} g}{4} \phi^{4}\right]\right) .
$$

Here, the integration measure is

$$
[d \phi]_{\Lambda}=\prod_{|k|<\Lambda} d \phi(k) .
$$

The renormalization is carried out as follows. We change from the scale $\Lambda$ to the scale $b \Lambda$, where $b<1$. The idea is to integrate over the degrees of freedom with large momenta. More precisely, we subdivide the integration variables $\phi(k)$ into two groups. The variables $\phi(k)$ with $b \Lambda \leq|k|<\Lambda$ are the degrees of freedom with large momenta, and we integrate over them. To distinguish these degrees of freedom, we define $\hat{\phi}(k)=\phi(k)$ for $b \Lambda \leq|k|<\Lambda$ and $\hat{\phi}(k)=0$ in other cases.

Then, we define new $\phi(k)$ such that they coincide with the old ones for $|k|<b \Lambda$ and are equal to zero for $|k|>b \Lambda$. Then, we can replace the old $\phi(k)$ in the original Lagrangian with $\phi+\hat{\phi}$ and rewrite Equation (2.4.1) in the form

$$
Z=\int[d \phi][d \hat{\phi}] \exp \left(-\int d^{d} x\left[\frac{1}{2}\left(\partial_{\mu} \phi+\partial_{\mu} \hat{\phi}\right)^{2}+\frac{m^{2}}{2}(\phi+\hat{\phi})^{2}+\frac{\gamma^{2} K \lambda}{\sqrt{2}}(\phi+\hat{\phi})^{3}+\frac{\gamma^{2} g}{4}(\phi+\hat{\phi})^{4}\right]\right),
$$

or

$$
\begin{gathered}
Z=\int[d \phi]\left[e ^ { - S ( \phi ) } \int [ d \hat { \phi } ] \operatorname { e x p } \left(-\int d^{d} x\left[\frac{1}{2}\left(\partial_{\mu} \hat{\phi}\right)^{2}+\frac{m^{2}}{2} \hat{\phi}^{2}+\frac{\gamma^{2} K \lambda}{\sqrt{2}}\left(3 \phi^{2} \hat{\phi}+3 \phi \hat{\phi}^{2}+\hat{\phi}^{3}\right)\right.\right.\right. \\
\left.\left.\left.+\frac{\gamma^{2} g}{4}\left(4 \phi^{3} \hat{\phi}+6 \phi^{2} \hat{\phi}^{2}+4 \phi \hat{\phi}^{3}+\hat{\phi}^{4}\right)\right]\right)\right] .
\end{gathered}
$$

In the last equation, the terms independent of $\hat{\phi}$ are collected in $S(\phi)$.

The quadratic terms of the form $\phi \hat{\phi}$ disappear because the Fourier components with different wave lengths are orthogonal. 
Let us transform (2.4.3) to the expression

$$
Z=\int[d \phi]_{b \Lambda} \exp \left(-\int d^{d} x S_{e f f}\right)
$$

where $S_{\text {eff. }}$ includes only the Fourier components $\phi(k)$ for which $|k|<b \Lambda$. $S_{\text {eff. }}$ equals $S(\phi)$ plus corrections proportional to powers of $\lambda$ and $g$.

To calculate the integrals over $\hat{\phi}(k)$, we use the following method. We assume that $m^{2}<<\Lambda^{2}$ and consider the term containing $\frac{1}{2} m^{2} \hat{\phi}^{2}$ as a perturbation even though this can be a coarse approximation.

The terms proportional to $\lambda$ and $g$ are also considered to be perturbations. Then, the leading term is

$$
\int S_{0}=\int_{b \Lambda \leq k \mid<\Lambda} \frac{d^{d} k}{(2 \pi)^{d}} \phi^{*}(k) k^{2} \phi(k)
$$

This term leads to the propagator

$$
K(k, p)=\frac{1}{k^{2}}(2 \pi)^{d} \delta^{d}(k+p) \Theta(k),
$$

where $\Theta(k)=1$ for $b \Lambda \leq|k|<\Lambda$ and is zero otherwise.

Proceeding in the conventional way, we obtain

$$
\int d^{d} x S_{e f f .}=\int d^{d} x\left[\frac{1}{2}(1+\Delta Z)\left(\partial_{\mu} \phi\right)^{2}+\frac{1}{2}\left(m^{2}+\Delta m^{2}\right) \phi^{2}+\frac{\gamma^{2} K}{\sqrt{2}}(\lambda+\Delta \lambda) \phi^{3}+\frac{\gamma^{2}}{4}(g+\Delta g) \phi^{4}+\Delta C\left(\partial_{\mu} \phi\right)^{4}+\Delta D \phi^{5}+\Delta E \phi^{6} \ldots\right] .
$$

To compare the new functional integral with the original one, we change the scale of distances and momenta by the rule

$$
k^{\prime}=\frac{k}{b}, \quad x^{\prime}=b x .
$$

In terms of the new variable, the action is written as

$$
\begin{gathered}
\int d^{d} x S_{e f f .}=\int d^{d} x^{\prime} b^{-d}\left[\frac{1}{2}(1+\Delta Z) b^{2}\left(\partial_{\mu}^{\prime} \phi\right)^{2}+\frac{1}{2}\left(m^{2}+\Delta m^{2}\right) \phi^{2}+\frac{\gamma^{2} K}{\sqrt{2}}(\lambda+\Delta \lambda) \phi^{3}\right. \\
\left.+\frac{\gamma^{2}}{4}(g+\Delta g) \phi^{4}+\Delta C\left(\partial_{\mu}^{\prime} \phi\right)^{4}+\Delta D \phi^{5}+\Delta E \phi^{6} \ldots\right] .
\end{gathered}
$$

In the course of the analysis, all the terms except for the first one were considered as small perturbations. As long as the original coupling constants are small, this is a correct approximation in the manipulations with effective Lagrangian (2.4.10). In our case, this corresponds to the low temperature limit.

The original functional integral gave propagator (2.4.7). The new action generates the same propagator if we change the field scale by the rule

$$
\phi^{\prime}=\left[b^{2-d}(1+\Delta Z)\right]^{1 / 2} \phi .
$$

Upon such a change, the unperturbed action takes the original form, and various perturbations are subject to the transformation

$$
\int d^{d} x S_{e f f .}=\int d^{d} x^{\prime}\left[\frac{1}{2}\left(\partial_{\mu}^{\prime} \phi^{\prime}\right)^{2}+\frac{1}{2} m^{\prime 2} \phi^{2}+\frac{\gamma^{2} K}{\sqrt{2}} \lambda^{\prime} \phi^{\prime 3}+\frac{\gamma^{2}}{4} g^{\prime} \phi^{\prime 4}++!^{\prime}\left(\partial_{\mu}^{\prime} \phi^{\prime}\right)^{4}+D^{\prime} \phi^{\prime 5}+\Delta E^{\prime} \phi^{\prime 6}\right] .
$$

Here, the new parameters of the Lagrangian are

$$
\begin{gathered}
m^{\prime 2}=\left(m^{2}+\Delta m^{2}\right)(1+\Delta Z)^{-1} b^{-2}, \\
\lambda^{\prime}=(\lambda+\Delta \lambda)(1+\Delta Z)^{-3 / 2} b^{\frac{d}{2}-3}, \\
g^{\prime}=(g+\Delta g)(1+\Delta Z)^{-2} b^{d-4}, \\
C^{\prime}=(C+\Delta C)(1+\Delta Z)^{-2} b^{d}, \\
D^{\prime}=(D+\Delta D)(1+\Delta Z)^{-5 / 2} b^{3 / 2 d-5},
\end{gathered}
$$




$$
E^{\prime}=(E+\Delta E)(1+\Delta Z)^{-3} b^{2 d-6},
$$

and so on.

In the original Lagrangian, we have $C=D=E=0$; however, if $C, D$ and $E$ were nonzero, the same equations would be valid.

The free field Lagrangian

$$
S_{0}=\frac{1}{2}\left(\partial_{\mu} \phi\right)^{2}
$$

is a fixed point of the renormgroup transformation.

In the neighborhood of $S_{0}$, we may neglect $\Delta m^{2}, \Delta \lambda, \Delta g$, and so on in the iterative equations (2.4.13) and retain only the terms that are linear in the perturbations. This yields the simple transformation

$$
m^{\prime 2}=m^{2} b^{-2}, \lambda^{\prime}=\lambda b^{\frac{d}{2}-3}, g^{\prime}=g b^{d-4}, C^{\prime}=C b^{d}, D^{\prime}=D b^{3 / 2 d-5}, E^{\prime}=E b^{2 d-6}, \text { etc. }
$$

Due to the use of the low temperature asymptotics, $\lambda$ and $g$ are small. Unfortunately, $m^{2}=1$ is not small; however, it is not large either. For that reason, we assume it to be small for convenience. As a result, for the one-dimensional case under consideration, we have

$$
\lambda^{\prime}=\lambda b^{-5 / 2}, g^{\prime}=g b^{-3}, m^{\prime 2}=m^{2} b^{-2} .
$$

Taking into account the fact that $b=\frac{\Lambda^{\prime}}{\Lambda}=\frac{L}{L^{\prime}}$ in (2.4.16), we obtain

$$
\frac{\lambda^{\prime}}{\lambda}=\left(\frac{L}{L^{\prime}}\right)^{-\frac{5}{2}}, \frac{g^{\prime}}{g}=\left(\frac{L}{L^{\prime}}\right)^{-3}
$$

This implies that

$$
\lambda \propto L^{\frac{5}{2}}
$$

and

$$
g \propto L^{3}
$$

Recall that $\lambda=\sqrt{T}$ and $g=T$. Then, (2.4.17) implies

$$
T \propto L^{5}
$$

and (2.4.19) implies

$$
T \propto L^{3} .
$$

Thus, we have obtained a dependence of the temperature of the stochastic term $T$ on the scale of the block-spin variables $L$; however, this dependence is contradictory. To resolve this contradiction, we apply the procedure for restoring the uniqueness of temperature described in Subsection 2.6.

\subsection{Renormalization of the Running Coupling Constants Using the MS Scheme in the One-dimensional Case}

We apply the MS scheme to the quantum field problem (2.3.14). In the process, we use the dimensionless coupling constants $\lambda$ and $g$, the dimensional scale of the imparted momentum $\mu$, and the dimensional bare constants $\lambda_{0}$ and $g_{0}$.

It is clear from Subsection 2.3 that we deal with the one-dimensional field theory. In fact, this is exactly quantum mechanics, in which there are no divergences and there is no need for renormalization when physical quantities are calculated. In addition, there is no problem of relation between different renormalization prescriptions, which is examined by the renormalization group.

As applied to the problem under consideration, the application of the renormalization group method in the form of the MS scheme is significantly facilitated.

The simplification is that when the renormalization is performed by the MS scheme, we use counterterms with zero coefficients multiplying the singular and finite terms.

Thus, we use the MS scheme with the dimensional regularization (Ramond, 1981; Collins, 1984; Weinberg, 2001; Peskin \& Schroeder, 1995) for renormalization. This approach is based on the introduction of an arbitrary parameter $\mu$ having the dimension of mass into the interaction Lagrangian, where $\mu$ is the normalizing momentum. 
In the framework of this scheme, the counterterms are expanded in Loran series in the parameter $n-d$, where $n$ is the actual dimension of the space, and $d$ describes its floating dimension.

In the dimensional regularization method, ultraviolet divergences appear in the form of poles of physical amplitudes at the point $d=n$. To eliminate the singularities due to these poles, the bare coupling constants $g_{B}^{l}(d)$, including masses, must also have similar poles in which the residuals are determined from the regularity conditions of the physical amplitudes as $d \rightarrow n$.

The bare coupling constants generally have nonzero dimensions $\Delta_{l}(d)$, which depend on the floating dimension of the space $d$ so that it is convenient to consider the dimensionless quantities $g_{B}^{l}(d) \mu^{-\Delta_{l}(d)}$, where $\mu$ is the floating scale with the dimension of energy (mass).

For example, the redefined bare coupling constant can be represented by the sum of the singular terms that are proportional to positive powers $v$ of $1 /(n-d)$. The coefficients $b_{v}$ of these terms are determined from the condition of singularity elimination in the physical amplitudes as $d \rightarrow n$. In addition, this sum contains a remainder term, which is analytic in $d$ for $d=n$. This remainder is identified with the dimensionless renormalized coupling constant $g^{l}(\mu, d)$ so that

$$
g_{B}^{l}(d) \mu^{-\Delta_{l}(d)}=g^{l}(\mu, d)+\sum_{v=1}^{\infty}(d-n)^{-v} b_{v}^{l}\left(g^{l}(\mu, d)\right) .
$$

In this particular case, the main Lagrangian is

$$
S_{b}=\frac{(\partial \phi)^{2}}{2}+\frac{m^{2} \phi^{2}}{2}+\frac{\gamma^{2} K}{\sqrt{2}} \lambda \mu^{\alpha} \phi^{3}+\frac{\gamma^{2}}{4} g \mu^{\beta} \phi^{4},
$$

and the counterterm Lagrangian is

$$
S_{c t}=\delta Z \frac{(\partial \phi)^{2}}{2}+\frac{\delta m^{2} \phi^{2}}{2}+\frac{\gamma^{2} K}{\sqrt{2}} \delta \lambda \phi^{3}+\frac{\gamma^{2}}{4} \delta g \phi^{4} .
$$

The counterterms are sought in the form

$$
\begin{gathered}
\delta \lambda=\mu^{\alpha} \sum_{j=1}^{\infty}(1-d)^{-j} a_{j}(\lambda, g, \mu), \\
\delta g=\mu^{\beta} \sum_{j=1}^{\infty}(1-d)^{-j} b_{j}(\lambda, g, \mu), \\
\delta m^{2}=m^{2} \sum_{j=1}^{\infty}(1-d)^{-j} c_{j}(\lambda, g, \mu), \\
\delta Z=\sum_{j=1}^{\infty}(1-d)^{-j} d_{j}(\lambda, g, \mu) .
\end{gathered}
$$

At $n=1$, quantum field theory turns into quantum mechanics, and the ultraviolet divergences disappear. Therefore, they do not appear in the form of poles of physical amplitudes at the point $d=1$ any more. Hence, the singular terms disappear from (2.5.1), and it turns into the formula

$$
g_{B}^{l}(d)=g^{l}(\mu, d) \mu^{\Delta_{l}(d)} .
$$

It can be shown (Collins, 1984) that $g_{B}^{l}(n)$ are invariant under the renormgroup transformations

$$
\mu d g_{B}^{l}(n) / d \mu=0 .
$$

Typically, the coupling constants $g_{B}^{l}(n)$ are calculated up to a certain power of the coupling constants $g^{l}$. In the case under consideration, the resultant formulas are exact due to the absence of singularity. As a consequence, the basic formula in our case is (2.5.8).

In our case, we have two running coupling constants; therefore, it is convenient to rewrite (2.5.8) and the formula for the mass (2.5.6) in the form

$$
\lambda_{0}=\mu^{\alpha} \lambda, g_{0}=\mu^{\beta} g, m_{0}^{2}=m^{2} .
$$


It remains to determine the parameters $\alpha$ and $\beta$, which are the dimensionalities of the bare coupling constants. To this end, we analyze the dimensions of the bare coupling constants. We also show that there are no divergences, which simplifies all the calculations. For that purpose, we consider the contributions to the proper energy, which is denoted by $\Sigma\left(p^{2}\right)$.

Consider the diagrams that are one-loop contributions to the proper energy in the theory with action (2.3.14). These diagrams are shown in Figure 1; they are written as

$$
\Sigma_{1}\left(p^{2}\right)=\frac{1}{2} \frac{9 \lambda^{2}}{(2 \pi)} \int d k \frac{1}{\left[k^{2}+m^{2}\right]\left[(p+k)^{2}+m^{2}\right]}-\frac{3}{2} \frac{g}{2 \pi} \int d k \frac{1}{\left[k^{2}+m^{2}\right]},
$$

where $\Sigma_{1}\left(p^{2}\right)$ is the one-loop contribution to the proper energy.

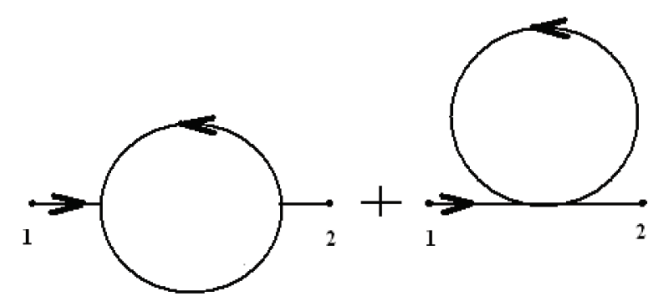

Figure 1. One-loop contribution to the proper energy

It is easy to verify that the one-loop contributions to the proper energy in the one-dimensional case are represented by converging integrals. The same is true of higher order contributions. Therefore, we do not need the singular parts of counterterms. Furthermore, if we are going to use the MS scheme, we do not need the finite parts of the counterterms either. Formally, we may set the coefficient multiplying those terms to zero.

Therefore, the bare masses and coupling constants are represented in terms of the renormalized masses and coupling constants in a very simple way; namely,

$$
\lambda_{0}=\mu^{\alpha} \lambda, g_{0}=\mu^{\beta} g, m_{0}^{2}=\mu^{2} m^{2} .
$$

Equation (2.5.12) can be solved directly without using the renormgroup equation.

Let us determine the dimensions of the bare coupling constants $\alpha$ and $\beta$. For this purpose, we need to analyze the dimensionalities. We assume that the dimension of mass is 1 , and we will denote the dimensions by braces; that is, $\{m\}=1$. The dimension of coordinate is -1 ; that is, $\{x\}=-1$ and $\left\{\frac{d}{d x}\right\}=1$.

Action is a dimensionless quantity; therefore, the dimension of the action density in the one-dimensional case is 1 .

Consequently, for the action density, we have

$$
\left\{\frac{1}{2}(\partial \phi)^{2}+\frac{m^{2}}{2} \phi^{2}+\frac{\gamma^{2} K \lambda}{\sqrt{2}} \phi^{3}+\frac{\gamma^{2} g}{4} \phi^{4}\right\}=1 .
$$

This implies that

$$
\left\{\frac{m^{2}}{2} \phi^{2}\right\}=\left\{\frac{1}{2}(\partial \phi)^{2}\right\}=2+2\{\phi\}=1 .
$$

Hence, $\{\phi\}=-\frac{1}{2}$.

Now, we can find the dimensions of the coupling constants $\lambda$ and $g$ from the equations

$$
\begin{aligned}
& \{\lambda\}+3\{\phi\}=\{\lambda\}-\frac{3}{2}=1, \\
& \{g\}+4\{\phi\}=\{g\}-2=1 .
\end{aligned}
$$

Hence, we obtain that $\{\lambda\}=\frac{5}{2}$ and $\{g\}=3$. Therefore, $\alpha=\frac{5}{2}$ and $\beta=3$. 
Recalling the definitions of $\lambda$ and $g$, we obtain the equations

$$
\frac{1}{\sqrt{\beta}}=\frac{1}{\sqrt{\beta_{0}}} \frac{1}{\mu^{\alpha}}, \frac{1}{\beta}=\frac{1}{\beta_{0}} \frac{1}{\mu^{\beta}} .
$$

By definition, $\beta$ is the reciprocal of temperature: $\beta=\frac{1}{T}$; therefore, the first formula in (2.5.17) implies that $T=T_{0} \mu^{-2 \alpha}$. The second formula in (2.5.17) implies that $T=T_{0} \mu^{-\beta}$. Unfortunately, these formulas give two different values for the temperature $T$ because $2 \alpha$ is different from $\beta$.

Thus, we have derived the equations for the unknown bare coupling constants

$$
\lambda_{0}=\lambda \mu^{\frac{5}{2}}, g_{0}=g \mu^{3} .
$$

They imply the following equations for the running coupling constants:

$$
\lambda=\frac{\lambda_{0}}{\mu^{5 / 2}}, g=\frac{g_{0}}{\mu^{3}} .
$$

Thus, using the MS scheme, we obtained the same result for the running coupling constants as was obtained by the Wilson technique (see formula (2.4.16)). Here, we faced the same difficulties. The point is that we have two coupling constants but only one temperature, and we obtain contradictory definitions of temperature from the two coupling constants.

Namely, we have

$$
T=T_{0} \mu^{-3}, T=T_{0} \mu^{-5} \text {. }
$$

Recalling that $\mu=\frac{1}{L}$, where $L$ is the scale of the block-spin variables, we obtain

$$
T=T_{0} L^{3}, T=T_{0} L^{5} .
$$

Thus we obtained the same contradictory dependence of the stochastic term temperature $T$ on the scale of the blockspin variables $L$ that was earlier obtained by the Wilson method $(2.4 .20,2.4 .21)$. To resolve this contradiction, we use the procedure for the restoration of the temperature uniqueness described in Subsection 2.6.

2.6 Procedure for the Restoration of the Unique Dependence of the Stochastic Term Temperature on the Scale of the Block-spin Variables and Determination of the Relation of this Temperature on the Scale of the Block-spin Variables

As a result of the renormalization of Lagrangian (2.3.14) by the Wilson technique and by the MS scheme, contradictory dependences of the temperature on the scale of the block-spin variables $(2.4 .40,2.4 .41)$ and $(2.5 .21)$ were obtained. The contradiction is that there are two coupling constants but only one temperature.

In addition, the bare constants $\lambda_{0}$ and $g_{0}$ are independent of the normalizing momentum $\mu$ by definition. However, if we choose one of temperature definitions (2.5.21), then one of the constants $\lambda_{0}$ or $g_{0}$ becomes dependent on $\mu$. To resolve this contradiction, we developed a procedure for restoring the uniqueness of temperature.

Let us see what will come out if we use the first definition of temperature $T=T_{0} \mu^{3}$. In this case, the bare coupling constant $\lambda_{0}$ becomes dependent on $\mu$, which contradicts its properties:

$$
\lambda_{0}=\mu^{5 / 2} \lambda=\mu^{5 / 2} \sqrt{\frac{T_{0}}{\mu^{3}}}=\mu \sqrt{T_{0}} .
$$

In this case, the bare constant $g_{0}$ is independent of $\mu$ :

$$
g_{0}=\mu^{3} g=\mu^{3} \frac{T_{0}}{\mu^{3}}=T_{0} .
$$

If we use the second definition of temperature $T=T_{0} \mu^{-5}$, then the bare coupling constant $\lambda_{0}$ is independent of $\mu$

$$
\lambda_{0}=\mu^{5 / 2} \lambda=\mu^{5 / 2} \sqrt{\frac{T_{0}}{\mu^{3}}}=\sqrt{T_{0}},
$$


while the bare coupling constant $g_{0}$ depends on $\mu$ :

$$
g_{0}=\mu^{3} g=\mu^{3} \frac{T_{0}}{\mu^{5}}=\frac{T_{0}}{\mu^{2}} .
$$

It is clear that, in any case, we can make only one of the two bare coupling constants invariant. The procedure for restoring the uniqueness of temperature is based on the fact that $\lambda_{0}$ and $g_{0}$ are independent of the normalizing momentum $\mu$.

The statistical sum Z, which was used in Subsection 2.3 for transforming the Malthus-Verhulst equation into the field problem, is the generating functional for the Green functions.

To develop the procedure for restoring the uniqueness of temperature, we use the connected two-point Green function $G\left(x_{2}-x_{1}, \lambda_{0}, g_{0}\right)$. It is represented in terms of the constants $\lambda_{0}, g_{0}$ and is, therefore, independent of the parameterization performed by the parameter $\mu$ (Collins, 1984).

Strictly speaking, it is the Green function multiplied by the coefficient $\zeta^{-2}$ related to the rescaling of the field operators that must be independent of the parameterization. However, this coefficient is equal to 1 for the reason indicated below. It is clear that we cannot make two objects $\lambda_{0}$ and $g_{0}$ independent of $\mu$ simultaneously, but we can make independent of $\mu$ a single object, namely, the connected two-point Green function $G$. To make it independent of $\mu$, we proceed as follows.

Since both definitions of the temperature $(2.4 .40,2.4 .41)$ and $(2.5 .21)$ cannot provide the invariance of the connected two-point Green function $G$, we use the general definition of the temperature in the form

$$
T=T_{0} f^{-1}(\mu)
$$

Then, both bare coupling constants cease to be invariant, and they take the form

$$
\lambda_{0}=\mu^{\frac{5}{2}} \lambda=\mu^{\frac{5}{2}} \sqrt{T}=\mu^{\frac{5}{2}} T_{0}^{\frac{1}{2}} f^{-\frac{1}{2}}, g_{0}=\mu^{3} g=\mu^{3} T=\mu^{3} T_{0} f^{-1} .
$$

The two-point function $G\left(x_{2}-x_{1}\right)$ depends only on the bare coupling constants; therefore, it is independent of $\mu$. This is because it must be independent of the parameterization determined by $\mu$. More precisely, it is the Green function multiplied by the coefficient $\zeta^{-2}$ related to the rescaling of the field operators that must be independent of the parameterization. However, this coefficient is equal to unity in the case under consideration due to the fact that the problem is one-dimensional. Due to the absence of divergences, the right-hand side of (2.5.7) vanishes. Therefore, the variation $\delta Z$ of the normalizing coefficient $Z$, which determines $\zeta$, also vanishes. Therefore, $Z$ remains invariant when $\mu$ varies. This yields $\zeta=1$. The reasoning above is implied by the formulas

$$
\begin{gathered}
G\left(x_{1}, \ldots, x_{N} ; \lambda(\mu), g(\mu), m(\mu), \mu\right)=\zeta^{-N}\left(x_{1}, \ldots, x_{N} ; \lambda^{\prime}\left(\mu^{\prime}\right), g^{\prime}\left(\mu^{\prime}\right), m^{\prime}\left(\mu^{\prime}\right), \mu^{\prime}\right), \\
\gamma=\frac{d}{d \mu} \ln Z=0
\end{gathered}
$$

in the one-dimensional case (Collins, 1984).

Here, $Z$ is a normalizing coefficient multiplying the term $\frac{(\partial \phi)^{2}}{2}$ in Lagrangian (2.3.14). The quantity $\gamma$ is the renormgroup coefficient corresponding to $Z$. The magnitude of $\gamma$ determines the coefficient $\zeta$

$$
\zeta\left(\mu^{\prime}, \mu\right)=\exp \left[-\frac{1}{2} \int_{\mu}^{\mu^{\prime}} \frac{d \mu^{\prime}}{\mu^{\prime}} \gamma\left(\left(\lambda\left(\mu^{\prime}\right), g\left(\mu^{\prime}\right)\right)\right]=1\right.
$$

for the one-dimensional case.

Let us replace the bare coupling constants $\lambda_{0}$ and $g_{0}$ in the connected two-point Green function with expressions (2.6.6) and require that the dependence of the connected two-point Green function on $\mu$ be as weak as possible. In this paper, we assume that it is independent of $\mu$ in the two first orders of the perturbation series in $\mu$. We perform the calculations in the simple so-called one-loop approximation. By definition, the two-point Green function is

$$
G\left(x_{2}-x_{1}\right)=\int[d \phi] \phi\left(x_{1}\right) \phi\left(x_{2}\right) e^{-S(\phi)},
$$


where the action $S(\phi)$ is

$$
S(\phi)=\int d x\left[\frac{1}{2} \phi_{x}^{2}+\frac{m_{0}^{2}}{2} \phi^{2}+\gamma^{2} K \frac{T_{0}^{1 / 2}}{\sqrt{2}} \mu^{5 / 2} f^{-1 / 2} \phi^{3}+\gamma^{2} \frac{T_{0}}{4} \mu^{3} f^{-1} \phi^{4}\right] .
$$

The two-point Green function $G\left(x_{2}-x_{1}\right)$ depends on the function $f$ determined by (2.6.5) and on the parameter $\mu$. We should select $f$ so as to make the dependence on $\mu$ as weak as possible. We will consider the low-temperature asymptotics, that is, the solution in region of small $\mu$. For small $\mu, F=\mu^{3}$ is a good zero-order approximation. Indeed, the action $S$ in this case takes the form

$$
S(\phi)=\int d x\left[\frac{\phi_{x}^{2}}{2}+m_{0}^{2} \frac{\phi^{2}}{2}+\gamma^{2} K \frac{T_{0}^{1 / 2}}{\sqrt{2}} \mu \phi^{3}+\gamma^{2} \frac{T_{0}}{4} \phi^{4}\right] .
$$

In the case of small $\mu, S$ is independent of $\mu$. Below, we find the first two corrections for the relation $\frac{1}{f}=\frac{1}{\mu^{3}}$. To simplify the calculations, we expand in the perturbation series the function $\frac{1}{f}$ :

$$
\frac{1}{f}=\left(\frac{1}{\mu^{3}}+\frac{\alpha_{1}}{\mu^{2}}+\frac{\alpha_{2}}{\mu}+\ldots .\right)
$$

The action $S$ takes the form

$$
S=S_{1}+S_{2}+S_{3}
$$

where

$$
\begin{gathered}
S_{1}=\int d x\left[\frac{\phi_{x}^{2}}{2}+m_{0}^{2} \frac{\phi^{2}}{2}+\gamma^{2} \frac{T_{0}}{4} \phi^{4}\right], \\
S_{2}=\int d x\left[\gamma^{2} K \frac{T_{0}^{1 / 2}}{\sqrt{2}} \mu\left(1+\alpha_{1} \mu+\alpha_{2} \mu^{2}+\ldots\right)^{1 / 2} \phi^{3}\right], \\
S_{3}=\int d x\left[\gamma^{2} K \frac{T_{0}}{4}\left(\alpha_{1} \mu+\alpha_{2} \mu^{2}+\ldots\right) \phi^{4}\right] .
\end{gathered}
$$

Next, we use the expansion

$$
\begin{gathered}
e^{-S_{2}}=1-\int d x\left[\gamma^{2} K \frac{T_{0}^{1 / 2}}{\sqrt{2}} \mu\left(1+\alpha_{1} \mu+\alpha_{2} \mu^{2}\right)^{1 / 2} \phi^{3}(x)\right]+ \\
+\frac{1}{2} \int d x \int d \bar{x}\left[\gamma^{4} K^{2} \frac{T_{0}}{2} \mu^{2}\left(1+\alpha_{1} \mu+\alpha_{2} \mu^{2}+\ldots\right) \phi^{3}(x) \phi^{3}(\bar{x})\right]+\ldots \\
e^{-S_{3}}=1-\int d x\left[\gamma^{2} \frac{T_{0}}{4}\left(\alpha_{1} \mu+\alpha_{2} \mu^{2}+\ldots .\right) \phi^{4}(x)\right]+\ldots
\end{gathered}
$$

In the first order in $\mu$, we have

$$
-\int[d \phi] \phi\left(x_{1}\right) \phi\left(x_{2}\right) \int d x\left[\gamma^{2} K \frac{T_{0}^{1 / 2}}{\sqrt{2}} \mu \phi^{3}(x)\right] e^{-S_{1}}-\int[d \phi] \phi\left(x_{1}\right) \phi\left(x_{2}\right) \int d x\left[\gamma^{2} \frac{T_{0}}{4}\left(\alpha_{1} \mu \phi^{4}(x)\right] e^{-S_{1}}=0 .\right.
$$

Since the first term on the left-hand side in (2.6.20) vanishes, we obtain $\alpha_{1}=0$. In the second order in $\mu$, we have

$$
-\int[d \phi] \phi\left(x_{1}\right) \phi\left(x_{2}\right) \int d x \int d \bar{x}\left[\gamma^{4} K^{2} \frac{T_{0}}{4} \mu^{2} \phi^{3}(x) \phi^{3}(\bar{x})\right] e^{-S_{1}}-\int[d \phi] \phi\left(x_{1}\right) \phi\left(x_{2}\right) \int d x\left[\gamma^{2} \frac{T_{0}}{4}\left(\alpha_{2} \mu^{2} \phi^{4}(x)\right] e^{-S_{1}}=0 .\right.
$$

Using (2.6.21), we can determine the coefficient $\alpha_{2}$. Let

$$
\begin{gathered}
\left.a_{0}=\gamma^{2} K^{2} \int[d \phi] \phi\left(x_{1}\right) \phi\left(x_{2}\right) \int d x \int d \bar{x} \phi^{3}(x) \phi^{3}(\bar{x})\right] e^{-S_{1}}, \\
b_{0}=\int[d \phi] \phi\left(x_{1}\right) \phi\left(x_{2}\right) \int d x \phi^{4}(x) e^{-S_{1}} .
\end{gathered}
$$


Then, formula (2.6.21) takes the form

$$
a_{0} \mu^{2}-b_{0} \alpha_{2} \mu^{2}=0 .
$$

The calculations are carried out in the one-loop approximation. In this case $S_{1}$ is replaced with $S_{0}$ :

$$
S_{0}(\phi)=\int d x\left[\frac{1}{2} \phi_{x}^{2}+\frac{m_{0}^{2}}{2} \phi^{2}\right] .
$$

Let

$$
\begin{gathered}
\left.a=\gamma^{2} K^{2} \int[d \phi] \phi\left(x_{1}\right) \phi\left(x_{2}\right) \int d x \int d \bar{x} \phi^{3}(x) \phi^{3}(\bar{x})\right] e^{-S_{0}}, \\
b=\int[d \phi] \phi\left(x_{1}\right) \phi\left(x_{2}\right) \int d x \phi^{4}(x) e^{-S_{0}} .
\end{gathered}
$$

In the one-loop approximation, formula (2.6.21) takes the form

$$
a \mu^{2}-b \alpha_{2} \mu^{2}=0
$$

Let us determine $a$ and $b$.

They are Feynman path integrals. They are corrections to the two-point Green functions for the field theory with the interactions $\phi^{3}$ and $\phi^{4}$, respectively. These corrections are made in the simple so-called one-loop approximation. They are proportional to the diagrams in Figure 1 and can be calculated in the standard way. In this paper, we consider the connected Green function; therefore, only connected diagrams are automatically distinguished in the one-loop approximation. Details can be found, for example, in (Zee, 2003; Peskin \& Schroeder, 1995; Itzykson \& Zuber, 1980). Furthermore, for the sake of brevity, we only present the ratio of $a$ to $b$, which we denote by $A=\frac{a}{b}$.

$$
\begin{gathered}
A=\frac{3 \gamma^{2} K^{2} m_{0}}{2}\left\{\frac{1}{\left(4 m_{0}^{2}+p^{2}\right) m_{0}^{4}}+\frac{6}{\left(4 m_{0}^{2}+p^{2}\right)^{2} m_{0}^{2}}+\right. \\
+\frac{12}{\left(4 m_{0}^{2}+p^{2}\right)^{2}} \frac{1}{|p| \sqrt{4 m_{0}^{2}+p^{2}}}\left[\operatorname { l n } \left(1+\sqrt{\left.\left.\left.1+\frac{4 m_{0}^{2}}{p^{2}}\right)-\ln \left(-1+\sqrt{1+\frac{4 m_{0}^{2}}{p^{2}}}\right)\right]\right\} .}\right.\right.
\end{gathered}
$$

Here, we took into account that the square of the bare mass is $m_{0}^{2}=\gamma^{2} K^{2}$.

Thus, we have obtained an expression for $A$ in the $p$-representation by calculating the Fourier integral of (2.6.26, 2.6.27).

Since the parameter $A$ equals $\frac{a}{b}$, Equation (2.6.28) takes the form

$$
\alpha_{2}=A
$$

In (2.6.30), we have the yet undetermined momentum $p$ appearing in the quantity $A(p)$ determined by (2.6.29). The momentum $p$ must be set equal to the characteristic scale of the momentum $\mu$. In the case of the low-temperature asymptotics, it can be assumed to be zero. Then, $A$ takes the form

$$
A(0)=\frac{3}{2 \gamma^{3} K^{3}} .
$$

As a result, we have the temperature $T$ of the stochastic term in the form

$$
T=T_{0} \frac{1}{\mu^{3}}\left(1+\frac{3 \mu^{2}}{2 \gamma^{3} K^{3}}\right) .
$$

Thus, formula (2.6.32) must be used instead of formulas (2.4.20, 2.4.21) and (2.5.21), which give an ambiguous value of the stochastic term temperature as dependent on the scale of the block-spin variables. Using this formula, one can determine how the temperature should be changed to compensate for the change in the scale of the blockspin variables. 


\section{Results}

The original Malthus-Verhulst equation with the stochastic term was reduced to a quantum field problem for the one-dimensional case. To determine the dependence of the stochastic term temperature on the scale of the blockspin variables, the latter problem was renormalized using the quantum field renormalization group (the Wilson technique and the MS scheme). As a result, the same dependence of the stochastic term temperature on the scale of the block-spin variables was obtained in both cases.

The Wilson technique turned out to be clearer because the extra degrees of freedom are removed directly in the process of renormalization. On the other hand, the MS scheme is technically simpler as applied to the onedimensional case.

The dependence of the stochastic term temperature on the scale of the block-spin variables obtained by both methods turned out to be the same but ambiguous. In both cases, two values for this dependence were obtained. In Subsection 2.6, a special procedure for restoring the unique dependence of the temperature (formula 2.6.23) was developed.

\subsection{Main Formulas}

In addition to restoring the uniqueness of temperature, the result of this paper makes it possible to answer two interrelated questions.

First question: How the temperature must be changed to compensate for the change in the scale of the block-spin variables?

Second question: How the temperature changes with the change in the scale of the block-spin variables?

The answers to these questions are related by the inverse transformation.

Formula (2.6.32) gives an answer to the first question. To answer the second question, the factor $\mu^{-3}\left(1+\frac{3 \mu^{2}}{2 \gamma^{3} K^{3}}\right)$ in (2.6.32) should be inversed. In order to find out how the temperature changes with the change in the scale of the block-spin variables, formula (3.1.1) must be used instead of (2.6.32):

$$
T=T_{0} \frac{\mu^{3}}{\left(1+\frac{3 \mu^{2}}{2 \gamma^{3} K^{3}}\right)} .
$$

It is easily seen that, in the case of small $\mu$, the temperature $T$ changes as $T_{0} \mu^{3}$.

\subsection{Calculation of the Correlation Length}

Based on the results obtained above, we can determine some properties of the two-point Green function. Using formula (3.1.1), we obtain an expression for the correlation length $\tau$. Let the two-point Green function $G(t)$ be

$$
G(t)=\int[d \phi][d \eta] \phi\left(t_{1}\right) \phi\left(t_{1}+t\right) e^{-\beta \int d t \eta^{2}(t)} \delta\left(\phi_{t}-\phi(1-\phi)-\eta(t)\right) .
$$

Here, by the expression $\delta\left(\phi_{t}-\phi(1-\phi)-\eta(t)\right)$ we mean construct (2.3.4).

Upon integration with respect to $\eta$, we obtain

$$
G(t)=\int[d \phi] \phi\left(t_{1}\right) \phi\left(t_{1}+t\right) e^{-\beta \int d t\left(\phi_{t}^{2}+\phi^{2}-2 \phi^{3}+\phi^{4}\right)} .
$$

Let the asymptotics of $G(t)$ as $|t| \rightarrow \infty$ be

$$
G(t) \approx C e^{-\frac{|t|}{\tau}},
$$

where the powers of $t$ are included into $C$. Here, $\tau$ is the correlation length. Then, the results obtained above imply that

$$
\frac{T}{T^{\prime}}=\left(\frac{\tau(T)}{\tau\left(T^{\prime}\right)}\right)^{3}\left(\frac{2 \gamma^{3} K^{3}+3 \tau^{2}\left(T^{\prime}\right)}{2 \gamma^{3} K^{3}+3 \tau^{2}(T)}\right) .
$$

Formula (3.2.4) implies that, for low $T$, it holds that

$$
\tau(T)=\tau\left(T_{0}\right)\left(\frac{T}{T_{0}}\right)^{\frac{1}{3}} .
$$


Formula (3.2.5) makes it possible to determine the correlation length in the case of low temperatures.

\subsection{Construction of Random Trajectories}

The procedure for restoring the unique dependence of the temperature of the stochastic term on the scale of the block-spin variables allows us to solve the following problem.

Consider a single random trajectory that is a solution to Equation (2.3.1) at the temperature $T_{1}$. This trajectory can be obtained, for example, by computer simulation. Then, given this trajectory, we can construct solutions for different temperatures $T_{2}$ by simply averaging over the cells of scale $\Lambda$. Here, $\Lambda$ is the relative scale of the cell, that is, the length $L_{2}$ measured in terms of $L_{1}$. Now, we show how the quantities $T_{1}, T_{2}$, and $\Lambda$ are related.

In (2.6.32), the value $T_{0}$ is not defined. Therefore, only the ratio of the temperatures $T_{2}$ and $T_{1}$ is known:

$$
\frac{T_{2}}{T_{1}}=\frac{\mu_{2}^{3}}{\mu_{1}^{3}} \frac{2 \gamma^{3} K^{3}+3 \mu_{1}^{2}}{2 \gamma^{3} K^{3}+3 \mu_{2}^{3}} .
$$

For low $T_{2}$ and $T_{1}$, we have

$$
\frac{T_{2}}{T_{1}}=\frac{\mu_{2}^{3}}{\mu_{1}^{3}}=\frac{L_{1}^{3}}{L_{2}^{3}}=\Lambda^{-3},
$$

where $\Lambda=\frac{L_{2}}{L_{1}}$ is the relative scale of the block-spin variables. This formula shows how $L_{2}$ should be selected in terms of $L_{1}$. Therefore, for low $T_{2}$ and $T_{1}$, we have

$$
T_{2}=T_{1} \Lambda^{-3}
$$

Similarly, from the ensemble of solutions corresponding to $T_{1}$, we can obtain the ensemble of solutions corresponding to $T_{2}$. For that purpose, we should average over the cells of the scale $\Lambda$. The quantity $\Lambda$ is determined from Equation (3.3.3) at low $T_{2}$ and $T_{1}$.

Furthermore, there may be cases where the data concerning the population of a certain species are not exactly described by Equation (2.3.1). For that reason, an ensemble of trajectories cannot be constructed by computer. However, if such an ensemble is obtained experimentally, one can construct an ensemble for another temperature. To this end, averaging over the cells of the scale $\Lambda$ should be performed.

This approach opens the way for the wider use of experimental data. However, it should be taken into account that certain experience, intuition, and heuristic considerations are required.

\section{Discussion}

The Malthus-Verhulst equation is a maximally simplified variant of a realistic biological model. Such a model becomes much more realistic upon the introduction of a stochastic term. Its study is a many-sided problem, and only one of the sides is considered in this paper. To investigate this model, a variant of the renormalization group is developed, which includes a procedure for restoring the unique dependence of the stochastic term temperature on the scale of the block-spin variables and the determination of the dependence of this temperature on the scale of the block-spin variables.

In the general case, the features of the method depend on the problem it is applied to. In this paper, the idea behind the method is illustrated using a specific example. Upon transformation, the Malthus-Verhulst equation with the stochastic term is reduced to a one-dimensional quantum field problem, which actually is a quantum mechanics problem. This transformation considerably simplifies the investigation scheme.

As the first step, two variants of the renormgroup method are applied to the original equation-the MS scheme and the Wilson technique. When the running coupling constants are renormalized by these methods, ambiguity in the dependence of the stochastic term temperature on the scale of the block-spin variables appears. To resolve this difficulty, a special procedure is developed based on the results obtained by the MS scheme and by the Wilson technique.

Each of these methods has specific features, advantages, and disadvantages. The concept of the block-spin variables is used in the Wilson technique, while the MS scheme uses the scale of the imparted momentum $\mu=\frac{1}{L}$. The MS scheme makes it possible to determine the relation between the scale of the block-spin variables and the temperature in a shorter way. It does not use the integration over the excess modes $p>\frac{1}{L}$, where $L$ is the chosen scale; neither does it use the concept of block-spin variables explicitly. 
The Wilson technique is more tedious, but it is also clearer. The simplicity and brevity of the MS scheme make it preferable in the one-dimensional case. The advantages of the MS scheme in the one-dimensional case would be even more obvious for more complex equations, for example, for the Lotka-Volterra equations. If the stochastic terms are added to these equations, the terms of the form $\phi_{t} \phi \psi$ appear in the interaction Lagrangian. The running coupling constants multiplying these terms are much easier to renormalize by the MS scheme than by the Wilson technique.

\section{Conclusions}

The simple mathematical biology equation-the Malthus-Verhulst equation with a stochastic term-is investigated using field theory methods.

The result obtained in this paper helps restore the uniqueness of temperature when field theory methods are applied to this equation. This result allows one to determine the dependence of the stochastic term temperature on the scale of the block-spin variables and take into account the stochastic phenomena in this mathematical biology equation. It is of interest to investigate other mathematical biology equations (such as Lotka-Volterra and other Volterra-type equations) with stochastic terms using modern mathematical techniques.

The investigation of the Malthus-Verhulst equation with a stochastic term performed in this paper adds to the known sides of the renormgroup method one more face. The author believes that the proposed method will be useful for solving other problems.

\section{References}

Adzhemyan, L. T., Antonov, N. V., \& Vasil'ev. A. N. (1996). Quantum field renormalization group in the theory of developed turbulence. Physics-Uspekhi (Advances in Physical Sciences), 39(12), 1193-1219. http://dx.doi.org/10.1070/PU1996v039n12ABEH000183

Basykin, A. D. (1998). Nonlinear dynamics of interacting populations. World scientific series on nonlinear science series A, 11. Singapore: World Scientific. http://dx.doi.org/10.1142/2284

Bibik, Yu. V., \& Sarancha, D. A. (2008). Renormgroup and discrete mappings for biological models with seasonality (Russian). Moscow: Dorodnicyn Computing Centre of RAS.

Collins, J. (1984). Renormalization: an introduction to renormalization, the renormalization group and the operator product expansion. Cambridge: Cambridge University Press.

Feigenbaum, M. J. (1978). Quantitative universality for a class of nonlinear transformations. Journal of Statistical Physics, 19(1), 25-52. http://dx.doi.org/10.1007/BF01020332

Feigenbaum, M. J. (1979). The universal metric properties of nonlinear transformations. Journal of Statistical Physics, 21(6), 669-706. http://dx.doi.org/10.1007/BF01107909

Gell-Mann, M., \& Low, F. (1954). Quantum electrodynamics at small distances. Physical Review, 95(5), 13001312. http://dx.doi.org/10.1103/PhysRev.95.1300

Itzykson, C., \& Zuber, J.-B. (1980). Quantum field theory. New York, NY: McGrav-Hill.

Kardar, M., Parisi, G., \& Zhang, Y.-C. (1986). Dynamic scaling of growing interfaces. Physical Review Letters, 56(9), 889-892. http://dx.doi.org/10.1103/PhysRevLett.56.889

Kerner, E. H. (1957). A statistical mechanics of interacting biological species. The Bulletin of Mathematical Biophysics, 19(2), 121-146. http://dx.doi.org/10.1007/BF02477883

Kerner, E. H. (1959). Further considerations on the statistical mechanics of biological associations. The Bulletin of Mathematical Biophysics, 21(2), 217-255. http://dx.doi.org/10.1007/BF02476361

Malthus, T. R. (1970). An essay on the principal of population. London: Penguin Books.

Peskin, M., \& Schroeder, D. (1995). An introduction to quantum field theory. Reading, Mass: Addison - Wesley.

Ramond, P. (1981). Field theory: A Modern primer. Reading, Mass: Benjamin.

Weinberg, S. (2001). The Quantum theory of fields (Vol. II). Modern Applications. Cambridge: Cambridge University Press.

Wilson., K. (1971). Renormalization group and critical phenomena. I. Renormalization group and the Kadanoff 
scaling picture. Physical Review B, 4(9), 3174-3183. http://dx.doi.org/10.1103/PhysRevB.4.3174

Wilson., K. (1971). Renormalization group and critical phenomena. II. Phase-Space cell analysis of critical behavior. Physical Review B, 4(9), 3184-3205. http://dx.doi.org/10.1103/PhysRevB.4.3184

Wilson, K., \& Kogut, J. (1974). The Renormalization group and the epsilon expansion. Physics Reports, 12C(2), 75-199.

Zee, A. (2003). Quantum field Theory in a nutshell. Princeton. N. J.: Princeton University Press. 